\title{
Gauged R-symmetry and its anomalies in 4D $N=1$ supergravity and phenomenological implications
}

\author{
I. Antoniadis, ${ }^{a, b, c}$ D.M. Ghilencea ${ }^{d, e}$ and R. Knoops ${ }^{e, f}$ \\ ${ }^{a}$ Albert Einstein Center for Fundamental Physics, Institute for Theoretical Physics, \\ University of Bern, 5 Sidlestrasse, CH-3012 Bern, Switzerland \\ ${ }^{b}$ LPTHE, Universite Pierre et Marie Curie, F-75252 Paris, France \\ ${ }^{c}$ Ecole Polytechnique, F-91128 Palaiseau, France \\ dTheoretical Physics Department, \\ National Institute of Physics and Nuclear Engineering (IFIN-HH), \\ Bucharest, MG-6 07r125, Romania \\ ${ }^{e}$ CERN Theory Division, \\ CH-1211 Geneva 23, Switzerland \\ ${ }^{f}$ Instituut voor Theoretische Fysica, KU Leuven, \\ Clestijnenlaan 200D, B-3001 Leuven, Belgium \\ E-mail: Ignatios.Antoniadis@cpht.polytechnique.fr, \\ Ghilencea@theory.nipne.ro, Rob.knoops@cern.ch
}

ABSTRACT: We consider a class of models with gauged $\mathrm{U}(1)_{R}$ symmetry in $4 \mathrm{D} \mathrm{N}=1$ supergravity that have, at the classical level, a metastable ground state, an infinitesimally small (tunable) positive cosmological constant and a $\mathrm{TeV}$ gravitino mass. We analyse if these properties are maintained under the addition of visible sector (MSSM-like) and hidden sector state(s), where the latter may be needed for quantum consistency. We then discuss the anomaly cancellation conditions in supergravity as derived by Freedman, Elvang and Körs and apply their results to the special case of a $\mathrm{U}(1)_{R}$ symmetry, in the presence of the Fayet-Iliopoulos term $(\xi)$ and Green-Schwarz mechanism(s). We investigate the relation of these anomaly cancellation conditions to the "naive" field theory approach in global SUSY, in which case $\mathrm{U}(1)_{R}$ cannot even be gauged. We show the two approaches give similar conditions. Their induced constraints at the phenomenological level, on the above models, remain strong even if one lifted the GUT-like conditions for the MSSM gauge couplings. In an anomaly-free model, a tunable, $\mathrm{TeV}$-scale gravitino mass may remain possible provided that the $\mathrm{U}(1)_{R}$ charges of additional hidden sector fermions (constrained by the cubic anomaly alone) do not conflict with the related values of $\mathrm{U}(1)_{R}$ charges of their scalar superpartners, constrained by existence of a stable ground state. This issue may be bypassed by tuning instead the coefficients of the Kahler connection anomalies $\left(b_{K}, b_{C K}\right)$.

KEYwords: Supergravity Models, Supersymmetric Effective Theories, Supersymmetry Breaking, Superspaces

ARXIV EPRINT: 1412.4807 


\section{Contents}

1 Introduction 1

2 Constructing models with gauged R-symmetry 3

2.1 The model: de Sitter ground state and TeV-gravitino mass from $\mathrm{U}(1)_{R} \quad 3$

2.1.1 The metastability of the ground state 5

2.2 A toy model with an additional field in the visible sector 6

2.3 A toy model with a $\mathrm{U}(1)_{R}$-charged field in the hidden sector 8

$\begin{array}{lll}3 & \mathrm{U}(1)_{R} \text { anomalies in supergravity and their field theory view } & 9\end{array}$

4 Application to MSSM-like models $\quad 12$

5 Conclusions $\quad 16$

$\begin{array}{ll}\text { A Gauged } \mathrm{U}(1)_{R} \text { and fields charges } & 17\end{array}$

B Cancellation of anomalies with a $\mathrm{U}(1)_{R} \times \mathrm{SM}$ group $\quad 19$

\section{Introduction}

The aim to obtain de Sitter vacua in $4 \mathrm{D} N=1$ supergravity with an infinitesimally small, positive (tunable) cosmological constant is a difficult task [1-9]. One possible attempt in this direction is to use models with a gauged R-symmetry $\left(\mathrm{U}(1)_{R}\right)[10-14]$. The models with anomalous $\mathrm{U}(1)$ gauge symmetries other than $\mathrm{U}(1)_{R}$ usually lead to anti-de Sitter (AdS) minimum with broken supersymmetry (SUSY) [15] or the positivity of their squared soft scalars masses is difficult to achieve [16, 17].

In $[18,19]$ a minimal toy model based on a $\mathrm{U}(1)_{R}$ gauge symmetry was studied. In its original version, the minimal field content of the model is the supergravity multiplet $\left(e_{\mu}^{i}, \psi_{3 / 2}\right)$ coupled to the gauge multiplet of $\mathrm{U}(1)_{R}$ and the (string) dilaton superfield $S$. The dilaton $S$ (of scalar component $s$ ) enables the presence of a shift symmetry, $S \rightarrow S-i c_{\mathrm{GS}} \Lambda$, with $c_{\mathrm{GS}}$ a real constant, as a consequence of the dual representation description in terms of two-index antisymmetric tensor for $\operatorname{Im}[s]$. This symmetry is gauged and $S$ is the only field that transforms non-linearly under it. Thus, the dilaton participates to the 4D Green-Schwarz (GS) mechanism. The only superpotential allowed by this symmetry is of the form $W(s)=a \exp (b s)$ with $a$ a real constant and $b<0$, with ${ }^{1}$ a Kahler potential $\mathcal{K}(s, \bar{s})=-2 \kappa^{-2} \ln (s+\bar{s})$.

With this minimal action and field content, one can show that there exists a ground state for a scalar field vev with $\alpha \equiv b\langle s+\bar{s}\rangle=-0.1833[18,19]$. This ground state thus

\footnotetext{
${ }^{1}$ The case $b<0$ is nonperturbative. The case $b>0$ is not considered in this work.
} 
depends on $b$ only, for a vanishing vacuum energy. There is an extremely mild dependence on $c_{\mathrm{GS}}$ as well, if one also demands an infinitesimally small positive (tunable) value of the cosmological constant. At the same time, the gravitino mass $m_{3 / 2} \sim \kappa^{-1}(a b)$ is near the $\mathrm{TeV}$ scale $^{2}$ (by tuning $a$ ) and the scale of supersymmetry breaking is that of gravity mediation $\sqrt{\left\langle F^{s}\right\rangle}=\left(m_{3 / 2} m_{P} /|b|\right)^{1 / 2} \mathrm{GeV}$ (by tuning $b$ ), with both $F$ and $D$ term breaking from the dilaton.

The purpose of this paper is to study further this model with $\mathrm{U}(1)_{R}$ gauge symmetry, at both classical and quantum levels. We investigate if these nice properties of the model can be maintained in the presence of additional states in the visible and hidden sectors that a more realistic model demands. This model is extended in the visible sector by the gauge and chiral multiplets of the minimal supersymmetric standard model (MSSM). The effect of adding states in the hidden sector that may be demanded for quantum consistency (like anomaly cancellation) is also investigated while trying to maintain a $\mathrm{TeV}$ gravitino mass. Particular attention is paid to the anomaly cancellation mechanism in supergravity with gauged $\mathrm{U}(1)_{R}$.

The plan of the paper is as follows. In section 2 we review the initial toy model and examine the metastability of the ground state. We then consider two cases: 1) the addition $\mathrm{U}(1)_{R}$-neutral superfield in the visible sector and 2) the case of a $\mathrm{U}(1)_{R^{-}}$-charged superfield in the hidden sector. We check under what conditions the nice properties of the initial ground state that we mentioned (like $m_{3 / 2} \sim \mathrm{TeV}$, etc) are maintained.

In section 3 we investigate the anomaly cancellation in supergravity with gauged $\mathrm{U}(1)_{R}$. A general study of anomaly cancellation conditions in supergravity with a gauge group $G \times$ U(1) and a Green-Schwarz mechanism(s) and Fayet Iliopoulos terms (FI) of the anomalous $\mathrm{U}(1)$ was presented in two interesting papers $[22,23]$ (see also $[24,25]$ ). We study the exact relation of these anomaly cancellation conditions to the "naive" field theory approach conditions in global SUSY (using Tr over charges and GS mechanism), for the special case of gauged $\mathrm{U}(1)_{R}$. Note that in global SUSY $\mathrm{U}(1)_{R}$ cannot be gauged [26]. The spectrum is that of minimal $4 \mathrm{D} \mathrm{N}=1$ supergravity extended by MSSM-like superfields with gauge group of Standard Model (SM) times $\mathrm{U}(1)_{R}$. This is an interesting check and a stand-alone result, independent of the rest of the paper.

In section 4 we apply these results to MSSM-like models. We assume that matter superfields have $\mathrm{U}(1)_{R}$-charges equal to $Q$, use the GS mechanism in the presence of $\mathrm{U}(1)_{R} \mathrm{FI}$ terms (which shift the fermions charges) and, notably, relax GUT-like unification condition. Even after doing so, the anomaly cancellation remains a strong parametric constraint. We then examine, in simple examples, if the gravitino mass remains tunable to $\mathrm{TeV}$ values. We show that the addition to the MSSM hidden sector of a $\mathrm{U}(1)_{R}$ charged superfield (of fermion component $\psi_{z}$ and charge $R_{z}$ ) does not solve this problem. This is because the $\mathrm{U}(1)_{R} \mathrm{cu}-$ bic anomaly-induced constraint on the R-charge $q_{z} \sim R_{z}-\xi$ of the scalar superpartner of $\psi_{z}$, modifies the ground state of the model, thus loosing its properties like $m_{3 / 2} \sim \mathrm{TeV}$. However, tuning the coefficients $\left(b_{K}, b_{C K}\right)$ of the Kahler connection anomalies or a more complicated hidden sector could avoid this issue. Finally, appendix A presents details on

\footnotetext{
${ }^{2}$ We use $\kappa=1 / m_{p}, m_{p}=M_{\text {Planck }} /(\sqrt{8 \pi})=2.4 \times 10^{18} \mathrm{GeV}$.
} 
deriving the $\mathrm{U}(1)_{R}$ charges of the fields, in the conformal compensator description, and appendix B reviews the "naive" flat-space field theory results for anomalies.

\section{Constructing models with gauged R-symmetry}

In this section we discuss models with a $\mathrm{U}(1)_{R}$ gauge symmetry in $4 \mathrm{D} \mathrm{N}=1$ supergravity [10-14] (for the Lagrangian see $[20,21]$ ). The goal is to understand better SUSY breaking, metastability of the ground state and to see if $m_{3 / 2}$ remains tunable to $\mathrm{TeV}$ values in such models. Consider a Kahler potential $\mathcal{K}$ and superpotential $W$ and define

$$
G=\kappa^{2} \mathcal{K}+\ln \left[\kappa^{6}|W|^{2}\right]
$$

Then the scalar potential of the model is $V=V_{F}+V_{D}$ with

$$
\begin{aligned}
V_{D} & =\frac{1}{2}(\operatorname{Re} f(s))^{-1 a b} D_{a} D_{b} \\
V_{F} & =\kappa^{-4} e^{G}\left[G^{i}\left(G^{-1}\right)_{i}^{k} G_{j}-3\right] \\
& =e^{\kappa^{2} \mathcal{K}}\left[\left(W^{i}+\kappa^{2} \mathcal{K}^{i} W^{\dagger}\right)\left(\mathcal{K}^{-1}\right)_{i}^{j}\left(W_{j}+\kappa^{2} \mathcal{K}_{j} W\right)-3 \kappa^{2}|W|^{2}\right]
\end{aligned}
$$

while the auxiliary fields $F^{i}$

$$
F^{i}=-\kappa^{-1} e^{G / 2}\left(G^{-1}\right)_{j}^{i} G^{j}=-e^{\kappa^{2} \mathcal{K} / 2} \frac{|W|}{W^{\dagger}}\left(\mathcal{K}^{-1}\right)_{j}^{i}\left(W^{j}+\kappa^{2} \mathcal{K}^{j} W^{\dagger}\right)
$$

in a standard notation; ${ }^{3} f$ is the gauge kinetic function, $D_{a}$ are the Killing potentials $[20,21]$

$$
D_{a} \equiv-i \mathcal{F}_{a}+i X_{a}^{j}(\phi) \mathcal{K}_{j}, \quad \mathcal{F}_{a}=-\frac{X_{a}^{j} W_{j}}{\kappa^{2} W}, \quad \Rightarrow \quad D_{a}=\frac{i X_{a}^{j}}{\kappa^{2} W}\left[W_{j}+\kappa^{2} \mathcal{K}_{j} W\right]
$$

where $X_{a}^{j}$ are Killing vectors, with $\delta \phi^{j}=\Lambda^{a} X_{a}^{j}(\phi)$ under a gauge transformation of parameter $\Lambda^{a}$. Also, the (gauge) covariant derivative is defined by ${ }^{4} D_{\mu} \phi^{j}=\partial_{\mu} \phi^{j}-X_{a}^{j} A_{\mu}$.

\subsection{The model: de Sitter ground state and TeV-gravitino mass from $\mathrm{U}(1)_{R}$}

In $[18,19]$ (also [27]) a class of metastable de Sitter vacua was discussed, which have a tunable (infinitesimally small) value of the cosmological constant and a $\mathrm{TeV}$ gravitino mass, based on a gauged $\mathrm{U}(1)_{R}$. We briefly review this model in this section. The spectrum consists of a chiral multiplet (dilaton $S$ ) and a gauge multiplet of $\mathrm{U}(1)_{R}$ in addition to the supergravity multiplet $\left(e_{\mu}^{i}, \psi_{3 / 2}\right)$. The chiral multiplet $S$ (dilaton) has a shift symmetry ( $c_{\mathrm{GS}}$ is a real constant)

$$
S \rightarrow S-i c_{\mathrm{GS}} \Lambda \text {. }
$$

\footnotetext{
${ }^{3}$ We use: $G^{i}=\partial G / \partial \phi_{i}^{\dagger}, G_{j}=\partial G / \partial \phi^{j}, G_{j}^{i}=\partial^{2} G / \partial \phi_{i}^{\dagger} \partial \phi^{j}, G_{j}^{i}=\mathcal{K}_{j}^{i} . \mathcal{K}_{j}=\partial \mathcal{K} / \partial \phi^{j}, \mathcal{K}^{j}=\partial \mathcal{K} / \partial \phi_{j}^{\dagger}$, $W_{i}=\partial W / \partial \phi^{i}$ and $W^{i}=\partial W^{\dagger} / \partial \phi_{i}^{\dagger}$; the index $i$ in $\phi^{i}$ labels all fields of the model, including the dilaton $s$.

${ }^{4}$ To establish our conventions, for an Abelian case we use $X^{j} \equiv-i q^{j} \phi^{j}$, therefore $\phi^{j} \rightarrow \exp \left(-i q^{j} \Lambda\right) \phi^{j}$ for a field $\phi^{j}$ of charge $q^{j}$ under a gauge transformation $A_{\mu} \rightarrow A_{\mu}+\partial_{\mu} \Lambda^{a}$, therefore $D_{\mu} \phi^{j}=\left(\partial_{\mu} \phi^{j}+i q^{j} A_{\mu}^{a}\right) \phi^{j}$.
} 
which is gauged. $\mathcal{K}$ is defined below while gauge invariance dictates the form of $W$

$$
\mathcal{K}=-2 \kappa^{-2} \ln (s+\bar{s}), \quad W=\kappa^{-3} a e^{b s},
$$

where $a, b$ are dimensionless constants. In string theory $b>0$ is considered nonphysical and $b<0$ corresponds to a nonperturbative superpotential. We assume $b<0$. The gauge kinetic function of $\mathrm{U}(1)_{R}$ is taken to be $f(S)=S$; the dilaton transforms non-linearly, then $X_{a}^{s}$ is field-independent, $X_{a}^{s}=-i c_{\mathrm{GS}}$, see eq. (2.4). The scalar potential is then, using eqs. (2.1) to (2.4)

$$
\begin{aligned}
V & =V_{F}+V_{D} \\
V_{D} & =\frac{\kappa^{-4}}{s+\bar{s}}\left[b c_{\mathrm{GS}}-\frac{2 c_{\mathrm{GS}}}{s+\bar{s}}\right]^{2} \\
V_{F} & =\kappa^{-4}|a|^{2} e^{b(s+\bar{s})}\left[\frac{b^{2}}{2}-\frac{2 b}{s+\bar{s}}-\frac{1}{(s+\bar{s})^{2}}\right]
\end{aligned}
$$

Also $\mathcal{F}_{a}=i b c_{\mathrm{GS}} \kappa^{-2} \equiv-i \xi \kappa^{-2}$ is the Fayet-Iliopoulos term. The auxiliary $F^{s}$ of $S$ is

$$
F^{s}=-\frac{\kappa^{2}}{2} e^{\kappa^{2} \mathcal{K} / 2}(s+\bar{s})^{2}|W|\left[b-\frac{2}{(s+\bar{s})}\right]=-\frac{\kappa^{-1}|a|}{2} e^{b(s+\bar{s}) / 2}[b(s+\bar{s})-2]
$$

Minimising the scalar potential and imposing a small positive value for it at the minimum point, $V_{\min }=\kappa^{-4} \epsilon_{0}$ where $\kappa^{-4} \epsilon_{0} \approx\left(10^{-3} \mathrm{eV}\right)^{4}$, give

$$
\frac{e^{-\alpha}}{\alpha} \frac{2(\alpha-6)}{\alpha^{2}-2 \alpha-2}=\frac{|a|^{2}}{b c_{\mathrm{GS}}^{2}}
$$

and respectively

$$
\frac{e^{-\alpha}}{\alpha} \frac{(-2)(\alpha-2)^{2}}{(\alpha-2)^{2}-6}+\frac{2 \epsilon_{0} e^{-\alpha} \alpha^{2} b^{-3}}{\left[(\alpha-2)^{2}-6\right] c_{\mathrm{GS}}^{2}}=\frac{|a|^{2}}{b c_{\mathrm{GS}}^{2}}, \quad \alpha \equiv b\langle s+\bar{s}\rangle
$$

The second term in the l.h.s. of eq. (2.10) can be neglected to a good approximation, so the existence of a minimum and a vanishing $V_{\min }\left(\epsilon_{0}=0\right)$ can both be fixed by one constraint on the set $\left\{a, b, c_{\mathrm{GS}}\right\}$ :

$$
\alpha \approx-0.1833, \quad \frac{|a|^{2}}{b c_{\mathrm{GS}}^{2}} \approx-50.6557
$$

Note that $\alpha$ fixes only the product $b\langle\operatorname{Re}[s]\rangle$, so any change of $b$ can in principle ${ }^{5}$ be compensated by that of Re $[s]$. A very small change of $c_{\mathrm{GS}}$ can adjust a small (positive) cosmological constant without impact on solution (2.11). The spectrum contains a gravitino of mass

$$
\left|m_{3 / 2}\right|=\left|\kappa^{2} e^{\kappa^{2} \mathcal{K} / 2} W\right|=\kappa^{-1}\left|\frac{a b}{\alpha}\right| e^{\alpha / 2} \sim 1 \mathrm{TeV} \quad \text { if } \quad|a b| \approx 200 \kappa \times(1 \mathrm{GeV})
$$

\footnotetext{
${ }^{5}$ This has an impact on the value of $1 / g_{s}^{2} \equiv \operatorname{Re}[s]=\alpha /(2 b)$ where $g_{s}$ is the $4 \mathrm{D}$ coupling.
} 
As shown above, a $\mathrm{TeV}$ gravitino mass is possible if a second tuning constraint is satisfied $|a b| \sim 8.3 \times 10^{-17}$, by tuning $a$. For example, $a \sim c_{\mathrm{GS}} \sim 10^{-13}$ with $b \sim \mathcal{O}\left(10^{-3}-10^{-2}\right)$.

The scale of SUSY breaking is found after rescaling $s \rightarrow s \kappa^{-1}$ and $F^{s} \rightarrow F^{s} \kappa^{-1}$ to restore their mass dimension (before this, $[s]=0,\left[F^{s}\right]=1$ ). This gives $\left\langle F^{s}\right\rangle \sim m_{3 / 2} m_{P} /|b|$ or $\sqrt{\left\langle F^{s}\right\rangle} \sim 10^{10} / \sqrt{|b|} \mathrm{GeV}$, so we have gravity mediation, with the mentioned values of $b$.

The mass of the dilaton $s$ is found

$$
\begin{gathered}
m_{s}^{2}=\frac{(s+\bar{s})^{2}}{4}\left\langle\partial_{s} \partial_{s} V\right\rangle=\frac{1}{4} \frac{\kappa^{-2} b^{3} c_{\mathrm{GS}}^{2} g(\alpha)}{\alpha^{3}\left(\alpha^{2}-2 \alpha-2\right)}, \\
g(\alpha) \equiv \alpha^{5}-8 \alpha^{4}+8 \alpha^{3}+44 \alpha^{2}-72 \alpha-24 .
\end{gathered}
$$

Under the aforementioned constraints for parameters $a, b, c_{\mathrm{GS}}$, the mass of the dilaton is of order $\mathcal{O}(1-100) \mathrm{TeV}$

$$
m_{s} \approx 15.42|b|^{3 / 2} c_{\mathrm{GS}} \kappa^{-1}
$$

Finally, the gauge field $C_{\mu}$ of $\mathrm{U}(1)_{R}$ "absorbs" the axion ( $\operatorname{Im}[s]$ ) and its mass is found after canonical normalisation of the gauge kinetic terms ${ }^{6}$

$$
m_{C_{\mu}}=\frac{2 c_{\mathrm{GS}} \kappa^{-1}}{\langle s+\bar{s}\rangle^{3 / 2}} \approx 25.48 \times|b|^{3 / 2} c_{\mathrm{GS}} \kappa^{-1}
$$

which is above the TeV scale for considered $a, b, c_{\mathrm{GS}}$.

To conclude by tuning $a, b, c_{\mathrm{GS}}$ one can ensure the existence of a ground state with a small positive cosmological constant, a TeV-mass of the gravitino and a scale of SUSY breaking similar to that in gravity mediation. These results for a toy model can be used to construct a more realistic model for SUSY breaking in supergravity with gauged $\mathrm{U}(1)_{R}$; however, parameters $a, b, c_{\mathrm{GS}}$ must then satisfy extra constraints like anomaly cancellation constraining $c_{\mathrm{GS}}$ (see later), with strong impact on the viability of the models (such as the values of the gravitino and soft terms masses).

\subsubsection{The metastability of the ground state}

The ground state we found is metastable, since there is another minimum $V_{\min }=0$ for a runaway solution at $\operatorname{Re}[s]=\infty$. We thus need to estimate the probability $(\Gamma)$ for the current vacuum $V_{\min }=\kappa^{-4} \epsilon_{0}>0$ to decay into the vacuum $V_{\min }=0$ along the $\operatorname{Re}[s]$ direction, through the potential barrier (figure 1 in $[18,19]$ ). This is to ensure the ground state is lived long-enough. This probability is (per unit of time and volume)

$$
\Gamma=\mathcal{A} e^{-\frac{\mathcal{B}}{\hbar}}(1+O(\hbar))
$$

where $\hbar$ is the reduced Planck constant (we set $\hbar=1$ ), and $\mathcal{A}$ and $\mathcal{B}$ depend on the model. The value of $\mathcal{A}$ plays a minor role in comparison with the exponential suppression; $\mathcal{B}$ is

\footnotetext{
${ }^{6}$ The "unusual" power of 3 in $m_{C_{\mu}}$ accounts for the fact that the mass is expressed in terms of Planck units and is consistent with the heterotic string result (where the power is 2) if expressed in terms of string scale.
} 
fixed by the Euclidean action of the instanton (bounce) solution $\left(S_{1}\right)$ which in the limit of a very small energy difference between the two minima is [28-30]

$$
\mathcal{B}=\frac{27 \pi^{2} S_{1}^{4}}{2 \epsilon_{0}^{3}}
$$

We redefine the field $s$ into (for standard kinetic terms; $s$ is dimensionless)

$$
\phi_{s}=\kappa^{-1} \log (s+\bar{s})
$$

$S_{1}$ is given by $[28-30]$

$$
S_{1}=\int d \phi_{s} \sqrt{2 \mathcal{V}\left(\phi_{s}\right)}=\kappa^{-3}|a| \mathcal{S}(b)
$$

where $\mathcal{V}$ is the potential of $\phi_{s}$ and

$$
\mathcal{S}(b)=\sqrt{2} \int_{\ln \left(\frac{\alpha}{b}\right)}^{\infty} d x\left[e^{b e^{x}}\left(\frac{b^{2}}{2}-2 b e^{-x}-e^{-2 x}\right)+\frac{e^{-x}}{b \zeta(\alpha)}\left(2 e^{-x}-b\right)^{2}\right]^{1 / 2}
$$

which can be computed numerically. $\zeta(\alpha)$ is given by the expression in the l.h.s. of eq. (2.10) (where one can set $\epsilon_{0}=0$ to a good approximation). If we interpret Re $(s)$ as the (inverse of the) 4 D coupling $\left(1 / g_{s}^{2}\right)$ to a GUT-like value, $\operatorname{Re}(s) \approx 25$, this fixes $b$ via $b=\alpha /\langle s+\bar{s}\rangle \approx$ $-3 \times 10^{-3}$. For this value of $b$ one finds $\mathcal{S} \approx 0.0124106$. By demanding that the gravitino mass be of order $\mathrm{TeV}$ (by tuning $a$, see previous section) one finds $\mathcal{B} \approx 10^{297}$. Therefore $\Gamma$ is extremely small (largely due to the small difference between the two minima); ${ }^{7}$ the ground state is long lived enough to use this model as a starting point for building realistic models, by adding physical fields, that have such ground state along this field direction.

\subsection{A toy model with an additional field in the visible sector}

We would like to preserve the nice features of the previous toy model and its ground state, while coupling the model to the visible sector. We thus add to the model one physical scalar field $(\phi)$ not charged $^{8}$ under $\mathrm{U}(1)_{R}$. A general way to couple the visible to the hidden sector while preserving the gauged $\mathrm{U}(1)_{R}$ is to consider

$$
\mathcal{K}=-2 \kappa^{-2} \ln (s+\bar{s})+\phi^{\dagger} \phi, \quad W=A(\phi) e^{b s}+B(\phi) .
$$

$W$ generalises the form in eq. (2.6). If $B(\phi) \neq 0$, this implies that the scalar field $\phi$ in the visible sector should have an R-charge, which we do not consider in this sub-section. So $B=0$ and

$$
W=A(\phi) e^{b s} .
$$

With this $W$, the scalar potential is then $V=V_{F}+V_{D}$, see eq. (2.2), where

$$
\begin{aligned}
V_{D} & =\frac{\kappa^{-4}}{s+\bar{s}}\left[b c_{\mathrm{GS}}-\frac{2 c_{\mathrm{GS}}}{s+\bar{s}}\right]^{2}, \quad \sigma_{s}=\frac{1}{2}[b(s+\bar{s})-2]^{2}-3 \\
V_{F} & =\frac{e^{\kappa^{2} \phi^{\dagger} \phi}}{(s+\bar{s})^{2}}\left[\kappa^{2} \sigma_{s}|W(\phi)|^{2}+\left|\nabla_{\phi} W(\phi)\right|^{2}\right]
\end{aligned}
$$

\footnotetext{
${ }^{7}$ Usually values of $\mathcal{B} \geq 400$ are regarded as metastable enough [31].

${ }^{8}$ Its fermionic spartner $\psi$ has however a $\mathrm{U}(1)_{R}$ charge $R_{\psi}=R_{\phi}+\xi / 2=\xi / 2$, see the appendix.
} 
and $\nabla_{\phi} W=\left(\partial / \partial \phi+\kappa^{2} \phi^{\dagger}\right) W$. The auxiliary fields $F^{s}, F^{\phi}$ of $s$ and $\phi$ are

$$
\begin{aligned}
& F^{s}=-e^{\kappa^{2} \mathcal{K} / 2}|W| \frac{(s+\bar{s})^{2}}{2} \kappa^{2}\left(b-\frac{2}{s+\bar{s}}\right) \\
& F^{\phi}=-e^{\kappa^{2} \mathcal{K} / 2} \frac{|W|}{W^{\dagger}}\left[\nabla_{\phi} W\right]^{\dagger}
\end{aligned}
$$

For phenomenological reasons, in viable models one would like to avoid SUSY breaking by the "visible" sector, so we demand $\left\langle F^{\phi}\right\rangle=0$ or

$$
\left\langle\nabla_{\phi} A(\phi)\right\rangle=0 .
$$

With $A(\phi)$ being analytical, one sees that the only possibility to respect this is to have $\langle\phi\rangle=0,\left\langle\partial_{\phi} A\right\rangle=0$. This gives

$$
\langle A(\phi)\rangle=\kappa^{-3} a .
$$

Therefore

$$
W=\left[\kappa^{-3} a+\tilde{W}(\phi)\right] e^{b s}, \quad\langle\tilde{W}(\phi)\rangle=0 .
$$

The ground state of the model is found by minimising $V$ w.r.t. $\phi$ and $s$

$$
\frac{\partial V}{\partial \phi}=0, \quad \frac{\partial V}{\partial s}=0
$$

The former is automatically respected with eq. (2.25), also given that $\phi$ is not R-charged. The second condition gives

$$
e^{\kappa^{2}\left\langle|\phi|^{2}\right\rangle+b\langle s+\bar{s}\rangle}\left\langle\rho_{s}\right\rangle|a|^{2}=c_{\mathrm{GS}}^{2}\left(b-\frac{6}{s+\bar{s}}\right),
$$

where

$$
\rho_{s}=\frac{1}{2}\left[b^{2}(s+\bar{s})^{2}-2 b(s+\bar{s})-2\right] .
$$

which is identical to (2.9) for $\langle\phi\rangle=0$. Thus the previous ground state in eq. (2.9) defined by $\langle s+\bar{s}\rangle=\alpha / b$ is preserved, with $\langle\phi\rangle=0$.

Further, using the notation $w_{\phi \phi} \equiv \partial^{2} \tilde{W}(\phi) / \partial \phi^{2}$, we find

$$
\begin{aligned}
\left\langle\partial_{\phi} \partial_{\phi} V\right\rangle & =\frac{e^{\alpha}}{\alpha^{2}} b^{2}\left(\left\langle\sigma_{s}\right\rangle+2\right) a^{\dagger} \kappa^{-1}\left\langle w_{\phi \phi}\right\rangle \\
\left\langle\partial_{\phi} \partial^{\bar{\phi}} V\right\rangle & =\frac{e^{\alpha}}{\alpha^{2}} b^{2}\left[\left(\left\langle\sigma_{s}\right\rangle+1\right)|a|^{2} \kappa^{-2}+\left|\left\langle w_{\phi \phi}\right\rangle\right|^{2}\right]
\end{aligned}
$$

while $\left\langle\partial_{\phi} \partial_{s} V\right\rangle=0$ following from $\left\langle F^{\phi}\right\rangle=0$. The eigenvalues of the above mass matrix give

$$
m_{ \pm}^{2}=\frac{e^{\alpha}}{\alpha^{2}} b^{2}\left[\kappa^{-2} a^{2}\left(\left\langle\sigma_{s}\right\rangle+1\right)+\left|w_{\phi \phi}\right|^{2} \pm\left(\left\langle\sigma_{s}\right\rangle+2\right) \kappa^{-1}\left|a^{\dagger} w_{\phi \phi}\right|\right]
$$

If $\kappa^{-1}|a| \gg\left|w_{\phi \phi}\right|, m_{ \pm} \sim m_{3 / 2}$ where $m_{3 / 2}$ remains equal to that found previously in eq. (2.12). Finally the mass of the fermion $\psi$, superpartner of $\phi$, is $m_{\psi}=w_{\phi \phi} e^{b\langle s\rangle} /(\langle s+\bar{s}\rangle)$. 
In conclusion, the guideline to construct a realistic model is this: replace the visible sector field $\phi$ by the MSSM superfields assumed to be $\mathrm{U}(1)_{R}$ neutral, just like the field $\phi$. Then the ground state with all previous benefits ( $\mathrm{TeV}$ gravitino mass, gravity mediated SUSY breaking etc) is preserved. ${ }^{9}$ Then from eq. (2.27) we conclude that a minimal realistic model has

$$
W=\left[\kappa^{-3} a+\tilde{W}_{\mathrm{MSSM}}\right] e^{b s}
$$

where $\tilde{W}_{\text {MSSM }}$ is the usual MSSM superpotential with superfields replaced by their scalar components. The Kahler potential will be similar to that considered here, with the contribution of $\phi$ replaced by that of the MSSM fields. The squared soft scalar masses of such model can be shown to be positive and close to the square of the gravitino mass $\left(\mathrm{TeV}^{2}\right)$.

\subsection{A toy model with a $\mathrm{U}(1)_{R^{-}}$-charged field in the hidden sector}

The model considered in the previous section is still too simple in that the hidden sector contains only the scalar field $s$ and a $\mathrm{U}(1)_{R}$ gauge boson and their superpartners. Ensuring the quantum consistency of the model (anomaly cancellation condition ${ }^{10}$ ) and maintaining at the same time the nice properties of the ground state ( $\mathrm{TeV}$-scale gravitino, etc) may demand the presence of additional $\mathrm{U}(1)_{R}$ charged fields in the hidden sector.

Let us then add an extra scalar field $z$ (and its superpartner) in the hidden sector, with a $\mathrm{U}(1)_{R}$ charge $q_{z}$, and examine when the ground state of the initial model can be maintained, together with its benefits. The visible sector scalar field $\phi$ remains $\mathrm{U}(1)_{R^{-}}$ neutral (while its superpartner acquires a $\mathrm{U}(1)_{R}$ charge). As a further step from eq. (2.21), we take

$$
\mathcal{K}=-2 \kappa^{-2} \ln (s+\bar{s})+\phi^{\dagger} \phi+z^{\dagger} z, \quad W=A(\phi, z) e^{b s} .
$$

where we omitted a possible addition of a function $B(z)$ in $W$ for simplicity and for preserving the basic properties of the model. From the invariance of the action under $\mathrm{U}(1)_{R}$, we find the fields transformations under $\mathrm{U}(1)_{R}$

$$
\begin{aligned}
s & \rightarrow s-i c_{\mathrm{GS}} \Lambda, & A(\phi, z) & \rightarrow A(\phi, z) e^{-i \beta \Lambda}, \\
z & \rightarrow z e^{-i q_{z} \Lambda}, & W(\phi, z) & \rightarrow W(\phi, z) e^{-i\left(c_{\mathrm{GS}} b+\beta\right) \Lambda}
\end{aligned}
$$

so that $\delta A=-i \beta A \Lambda$ and with $\delta A=\partial_{z} A \delta z=-i q_{z} z \Lambda \partial_{z} A$ then $\partial \ln A / \partial \ln z=\beta / q_{z}$. Thus $A(\phi, z)=(\kappa z)^{\beta / q_{z}}\left[a \kappa^{-3}+\tilde{W}(\phi)\right]$, where $a$ is a constant and $W=(\kappa z)^{\beta / q_{z}}\left[a \kappa^{-3}+\right.$ $\tilde{W}(\phi)] e^{b s}$. The scalar potential is then $V=V_{F}+V_{D}$ with

$$
\begin{aligned}
& V_{D}=\frac{\kappa^{-4}}{s+\bar{s}}\left[\beta+\kappa^{2} q_{z}|z|^{2}+b c_{\mathrm{GS}}-\frac{2 c_{\mathrm{GS}}}{s+\bar{s}}\right]^{2} \\
& V_{F}=e^{\kappa^{2} \mathcal{K}}\left[\kappa^{2} \sigma_{s}|W|^{2}+\left|W_{\phi}+\kappa^{2} \phi^{\dagger} W\right|^{2}+\left|W_{z}+\kappa^{2} z^{\dagger} W\right|^{2}\right],
\end{aligned}
$$

\footnotetext{
${ }^{9}$ Such a case is discussed in section 4 .

${ }^{10}$ This is discussed in section 3.
} 
The Fayet-Iliopoulos term is now $\mathcal{F}_{a}=i\left(\beta+b c_{G S}\right) \kappa^{-2} \equiv-i \xi \kappa^{-2}$. Again we demand that $^{11}\left\langle F^{\phi}\right\rangle=0$ or $\left\langle\nabla_{\phi} W\right\rangle=0$ which is satisfied for a standard $\tilde{W}$, polynomial in fields (such as that of the MSSM) and $\langle\phi\rangle=0$.

The minimum condition of the potential w.r.t. $\phi$ is automatically satisfied since $\phi$ does not break SUSY. To simplify the analysis we consider that the scalar field $z$ does not enter in the superpotential, so $\beta=0$. This choice is also motivated by the analysis in the next section and impacts on the value of FI term $\xi$, giving $\xi=-b c_{\mathrm{GS}}$ as we had before. ${ }^{12}$ Then

$$
W=\left[a \kappa^{-3}+\tilde{W}(\phi)\right] e^{b s}
$$

The minimum conditions, evaluated at $\langle\phi\rangle=0$ give

$$
\begin{aligned}
e^{\gamma+\alpha} b|a|^{2}\left(\left\langle\rho_{s}\right\rangle+\gamma\right)(1-2 / \alpha)-\left[b c_{\mathrm{GS}}(1-2 / \alpha)+\gamma q_{z}\right]\left[b c_{\mathrm{GS}}(1-6 / \alpha)+\gamma q_{z}\right] & =0 \\
{\left[e^{\gamma+\alpha}(b / \alpha)|a|^{2}\left(\left\langle\sigma_{s}\right\rangle+1+\gamma\right)+2 q_{z}\left[q_{z} \gamma+b c_{\mathrm{GS}}(1-2 / \alpha]\right]\left\langle z^{\dagger}\right\rangle(b / \alpha)\right.} & =0
\end{aligned}
$$

where $\gamma=\kappa^{2}|\langle z\rangle|^{2}$ and with $\alpha=b\langle s+\bar{s}\rangle,\left\langle\sigma_{s}\right\rangle=(1 / 2)(\alpha-2)^{2}-3,\left\langle\rho_{s}\right\rangle=(1 / 2)\left(\alpha^{2}-2 \alpha-2\right)$. The condition $V_{\min }=\kappa^{-4} \epsilon_{0}=\left(10^{-3} \mathrm{eV}\right)^{4}$ gives

$$
e^{\alpha+\gamma}|a|^{2}(b / \alpha)\left(\left\langle\sigma_{s}\right\rangle+\gamma\right)+\left[q_{z} \gamma+b c_{\mathrm{GS}}(1-2 / \alpha)\right]^{2}=\epsilon_{0} \alpha / b
$$

The system of eqs. (2.38), (2.39) should be solved for $\alpha$ and $\gamma$ in terms of the parameters of the model. One can see that $\langle z\rangle=0$ is a solution to (2.39) which if used in eqs. (2.38), (2.40) gives two equations identical to $(2.9),(2.10)$ and thus have the same solution $\alpha=-0.1833$ as found there. With this value of $\alpha$, the square bracket in eq. (2.39) is positive and nonzero if $q_{z}<0$ and $c_{\mathrm{GS}}>0$. In this situation, the original ground state $\langle s+\bar{s}\rangle=\alpha / b,(b<0)$ together with $\langle\phi\rangle=\langle z\rangle=0$ is indeed a solution of this extended model provided that $q_{z}<0, c_{\mathrm{GS}}>0$. If these conditions are violated, then $\langle z\rangle \neq 0$ and the original vev for $s$ and its properties are not maintained. It can be shown that this solution is a ground state (i.e. local minimum) if a higher order Kahler term with $(\partial \mathcal{K} / \partial z)_{o}=0$ is added to $\mathcal{K}$, without modifying this solution.

In conclusion, a hidden sector field with charge $q_{z}<0$ can be added to the model of previous section without altering the vacuum of the initial theory, with the new field directions having vanishing vev's. As a result, $m_{3 / 2}$ is the same as found before (independent of $q_{z}$ ). Anomaly cancellation to which the superpartner fermion of $z$ will contribute can constrain $q_{z}$. We shall then check if $q_{z}<0, c_{\mathrm{GS}}>0$ are consistent with anomaly cancellation (section 4).

\section{$3 \mathrm{U}(1)_{R}$ anomalies in supergravity and their field theory view}

The discussion so far was at the classical level. Quantum consistency like anomalies cancellation is a strong constraint, discussed next. This can change the results we found, such as the $\mathrm{TeV}$-values for $m_{3 / 2}$, because the parameters of the models ( $a, b, c_{\mathrm{GS}}$ or charges) are

\footnotetext{
${ }^{11} F^{s}$ and $F^{\phi}$ are those of $(2.24)$ while $F^{z}=\left.F^{\phi}\right|_{\phi \rightarrow z}$.

${ }^{12}$ This is important since $\xi$ is related to the R-charges of the fields and plays a role in anomaly cancellation.
} 
more constrained now. This section is also relevant on its own, independent of the rest of the paper.

Anomaly cancellation in supergravity with an anomalous U(1) with Fayet-Iliopoulos term(s) and Green-Schwarz mechanism where discussed in the past in a general setting in $[22,23]$ that we use below. However, the relation and agreement of such results to the "naive" field theory approach was not examined for the rather special case of a gauged $\mathrm{U}(1)_{R}$. In fact in global SUSY $\mathrm{U}(1)_{R}$ cannot even be gauged. In this section we carefully investigate this relation. We refer the reader to these two papers for the details of the supergravity analysis while in appendix B we review the naive field theory results (flat space), with our conventions for the $\mathrm{U}(1)_{R}$ charges defined in appendix A. For a previous detailed study see [11].

The field content is that of minimal supergravity, with gauged $\mathrm{U}(1)_{R}$ and the dilaton $S$ for the GS mechanism, as in previous sections, and with additional (say MSSM-like) superfields and SM gauge group times $\mathrm{U}(1)_{R}$. Anomaly cancellation conditions for the cubic anomaly $\mathrm{U}(1)_{R}^{3}$ ((a) below) and the mixed anomalies of $\mathrm{U}(1)_{R}$ with the Kahler connection $K_{\mu}((\mathrm{b}),(\mathrm{c}))$, with the SM gauge group (d) and gravity (e) were found in eqs. (4.4) in section 4 of [22]. These are

$$
\begin{aligned}
\text { (a) } & \mathrm{C} \tilde{\mathrm{C}}: & 0 & =\operatorname{Tr}\left[(Q+\xi / 2) Q^{2}\right]+\xi a_{K C C}-c_{\mathrm{GS}} b_{C} \\
(b) & C \tilde{K}: & 0 & =\operatorname{Tr}[(Q+\xi / 2) Q]-\xi a_{C K K}-a_{K C C}-c_{\mathrm{GS}} b_{C K} \\
(c) & K \tilde{K}: & 0 & =\operatorname{Tr}[Q]-\frac{1}{2} \xi\left(n_{\lambda}+3-n_{\psi}\right)+4 a_{C K K}-4 c_{\mathrm{GS}} b_{K} \\
(d) & (F \tilde{F})_{\alpha}: & 0 & =-\operatorname{Tr}\left[Q\left(\tau^{a} \tau^{b}\right)_{\alpha}\right]+\frac{\xi}{2}\left[C_{2}(G)-C(r)\right]_{\alpha} \delta^{a b}+\frac{1}{3} c_{\mathrm{GS}} b_{A, \alpha} \delta^{a b} \\
(e) & \mathcal{R} \tilde{\mathcal{R}}: & 0 & =\operatorname{Tr}[Q]-\frac{\xi}{2}\left(n_{\lambda}-21-n_{\psi}\right)+8 c_{\mathrm{GS}} b_{R}
\end{aligned}
$$

where labels the dual field strength. $C_{\mu}\left(C_{\mu \nu}\right)$ is the gauge field (strength) of $\mathrm{U}(1)_{R}$; $F_{\mu \nu}$ is a field strength corresponding to gauge fields $A_{\mu}$ of the SM gauge group (with group generators $\left.{ }^{13} \tau^{a}\right) ; \alpha$ is a group index that runs over $\mathrm{U}(1)_{Y}, \mathrm{SU}(2)_{L}, \mathrm{SU}(3) . K_{\mu}$ is the Kahler connection that essentially fixes the coupling of the gravitino. These three fields are involved in conditions (a), (b), (c), (d). Condition (e) is for the mixed, $\mathrm{U}(1)_{R^{-} \text {-gravitational }}$ anomaly. There is also the usual SM condition $\operatorname{Tr}\left[\tau^{a}\left\{\tau^{b}, \tau^{c}\right\}\right]=0$ for SM group generators.

Regarding notation, $n_{\lambda}$ is the number of gauginos present, $n_{\psi}$ is the number of Weyl fermions (matter fermions and dilatino), $Q$ 's are the $\mathrm{U}(1)_{R}$ charges of matter superfields and $\xi$ is the Fayet Iliopoulos term of our $\mathrm{U}(1)_{R}$, (see also appendix A). Unlike other types of anomalous $\mathrm{U}(1)$ 's, for the special case of $\mathrm{U}(1)_{R}$, the $\mathrm{SM}$ gauginos, $\mathrm{U}(1)_{R}$ gaugino and gravitino are all charged under $\mathrm{U}(1)_{R}$ and contribute to the anomalies. Also $\operatorname{Tr}_{r}\left(\tau^{a} \tau^{b}\right)=$ $C(r) \delta^{a b}$ is the trace over the irreducible representations $r$, and $f^{a c d} f^{b c d}=\delta^{a b} C_{2}(G)$. The terms in eqs. (3.1) proportional to $\xi$ of coefficients +3 and -21 denote the contributions of the gravitino to those anomalies [32]. Finally, $c_{\mathrm{GS}}$ is the GS coefficient and $a_{K C C}, a_{C K K}$ are coefficients of local counterterms that can be present (defined shortly).

\footnotetext{
${ }^{13}$ Refs. [22, 23] use anti-hermitian $T^{a}=-i \tau^{a}$, so we added a minus in front of $T r$ in line (d) of eqs. (3.1).
} 
To understand the role of Kahler connection $K_{\mu}$ it is instructive to write down the gauge $\left(V_{\mu}\right)$ couplings, present, in addition to the spin-connection, in the covariant derivatives of the gravitino $\psi_{3 / 2}$, gauginos $\lambda$ and matter fermions $\psi$ (such as those in the MSSM): ${ }^{14}$

$$
\begin{aligned}
\psi_{3 / 2}: & V_{\mu} & =-(i / 2) K_{\mu} \\
\lambda^{a}: & V_{\mu}^{a b} & =-A_{\mu}^{c} f^{a b c}-(i / 2) K_{\mu} \delta^{a b} \\
\psi^{\alpha}: & V_{\mu} & =A_{\mu}^{a}\left(-i \tau^{a}\right)+i Q C_{\mu}+(i / 2) K_{\mu}
\end{aligned}
$$

The local counterterms that come with coefficients $a_{K C C}, a_{C K K}$ are [22, 23]

$$
\delta \mathcal{L}_{1}=\frac{1}{24 \pi^{2}} \epsilon^{\mu \nu \rho \sigma}\left[a_{C K K} C_{\mu} K_{\nu} \partial_{\rho} K_{\sigma}+a_{K C C} K_{\mu} C_{\nu} \partial_{\rho} C_{\sigma}\right]
$$

and $b_{C}, b_{C K}, b_{K}$, etc, of eqs. (3.1) are coefficients present in the Chern-Simons terms

$$
\begin{aligned}
\delta \mathcal{L}_{2}= & \frac{1}{48 \pi^{2}} \operatorname{Im}[s] \epsilon^{\mu \nu \rho \sigma} \partial_{\mu} \Omega_{\nu \rho \sigma} \\
= & \frac{1}{48 \pi^{2}} \operatorname{Im}[s]\left[b_{C} C_{\mu \nu} \tilde{C}^{\mu \nu}+b_{C K} C_{\mu \nu} \tilde{K}^{\mu \nu}+b_{K} K_{\mu \nu} \tilde{K}^{\mu \nu}\right. \\
& \left.\quad+b_{A, \alpha}\left(F_{\mu \nu} \tilde{F}^{\mu \nu}\right)_{\alpha}+b_{R} \mathcal{R}_{\mu \nu} \tilde{\mathcal{R}}^{\mu \nu}\right]
\end{aligned}
$$

with dual field definition $\tilde{C}^{\mu \nu}=1 / 2 \epsilon^{\mu \nu \rho \sigma} C_{\rho \sigma}$, etc. Some of the coefficients $b_{C}, b_{K}, b_{C K}$, $b_{R}$ can be related, as for example in heterotic string theory (for $b_{C}, b_{R}$ ) and cannot be adjusted at will. However, this applies only to anomalous U(1)'s which are not of $R$-type, since it is difficult to derive $\mathrm{U}(1)_{R}$ from strings. We relax this constraint and consider them independent.

It is assumed that the dilaton $(S)$ is the only (super)field that transforms non-linearly under the gauged $\mathrm{U}(1)_{R}$ and thus it implements the Green-Schwarz mechanism. The canonical normalization of the gauge kinetic term for $\mathrm{U}(1)_{R}$ gauge field $\left(C_{\mu}\right)$ gives $b_{C}=$ $12 \pi^{2}$ and we assume that this is the case in the following, while $b_{K}$ and $b_{C K}$ can in general be non-zero.

Supergravity conditions (3.1) are not transparent from the "naive" field theory point of view for the anomalies of $\mathrm{U}(1)_{R}$. So let us clarify the link of these conditions to the field theory result in appendix B. First, the $\mathrm{U}(1)_{R}$ charges of the fields are shown below (see appendix A) and depend on the FI term(s):

$$
R_{\psi}=Q+\xi / 2, \quad R_{\lambda}=R_{\psi_{3 / 2}}=-\xi / 2, \quad R_{\psi_{s}}=\xi / 2 .
$$

where $Q$ is the charge of the superfield or scalar superpartner of $\psi ; R_{\lambda}, R_{\psi_{3 / 2}}$ and $R_{\psi_{s}}$ are the charges of the gaugino $\lambda$, gravitino $\psi_{3 / 2}$ and dilatino $\psi_{s}$, respectively. Using this information, the first three relations in eqs. (3.1) can be combined, after multiplying them by $4,4 \xi$ and $\xi^{2}$ respectively and then adding them and using that $\operatorname{Tr} 1=n_{\psi}-1$. The result is ${ }^{15}$

$$
\operatorname{Tr}\left[R_{\psi}^{3}\right]+n_{\lambda} R_{\lambda}^{3}+3 R_{\psi_{3 / 2}}^{3}+R_{\psi_{s}}^{3}=c_{\mathrm{GS}}\left[b_{C}+\xi b_{C K}+\xi^{2} b_{K}\right]
$$

\footnotetext{
${ }^{14}$ Note that eqs. (3.2) apply for the case of flat field-space.

15 "- 1 " in $\operatorname{Tr} 1=n_{\psi}-1$ isolates the dilatino from matter fermions $\psi$ (the charge $Q$ of the dilaton is 0 .).
} 
One recognizes in the l.h.s. the usual field theory cubic $\mathrm{U}(1)_{R}^{3}$ anomaly cancellation condition in the presence of FI terms and GS mechanism, in which all fermionic contributions are added and compensated by a GS shift in the rhs: the trace adds all matter fermions contributions, $n_{\lambda}$ is the number of gauginos ${ }^{16}$ each of a contribution $R_{\lambda}^{3} ;(+3) R_{\psi_{3 / 2}}^{3}$ is the gravitino contribution, three times larger than that of one gaugino [32]. The result above has (with $b_{C}=12 \pi^{2}$ and $b_{C K}=b_{K}=0$ ) the same form as the "naive" field theory result, eqs. (B.5), (B.7) in appendix B. This is interesting since in global SUSY U $(1)_{R}$ cannot even be gauged. Note however the difference in the r.h.s. due to $\xi b_{C K}+\xi^{2} b_{K}$. The terms in $\delta \mathcal{L}_{2}$ of coefficients $b_{K}, b_{C K}$ are not present in naive field theory case, and give extra freedom in canceling this anomaly.

The two remaining independent conditions of constraints (a), (b), (c) in eq. (3.1), refer to Kahler and mixed $\mathrm{U}(1)_{R}$-Kahler connection. They can always be respected by a suitable choice of $a_{K C C}$ and $a_{C K K}$ of the local counterterms shown and are not discussed further. One finds (by combining these two remaining constraints with condition (e) in eq. (3.1)), that $a_{C K K}=3 \xi+c_{\mathrm{GS}}\left(b_{K}+2 b_{R}\right)$, while $a_{K C C}$ is found from one of equations (a), (b), (c) in eq. (3.1).

The last two conditions in eq. (3.1) can be re-written as

$$
\begin{array}{rrr}
(F \tilde{F})_{\alpha}: & \operatorname{Tr}\left[R_{\psi}\left(\tau^{a} \tau^{b}\right)_{\alpha}\right]+C_{2}\left(G_{\alpha}\right) \delta^{a b} R_{\lambda}=(1 / 3) c_{\mathrm{GS}} b_{A, \alpha} \delta^{a b} \\
\mathcal{R} \tilde{\mathcal{R}}: & \operatorname{Tr}\left[R_{\psi}\right]+n_{\lambda} R_{\lambda}+(-21) R_{\psi_{3 / 2}}+R_{\psi_{s}}=-8 c_{\mathrm{GS}} b_{R}
\end{array}
$$

where $C_{2}(G) \delta^{a b}=f^{a c d} f^{b c d}$ with $C_{2}(G)=N$ for $\mathrm{SU}(N)$ and 0 for $\mathrm{U}(1), \alpha$ labels the groups $\mathrm{U}(1)_{Y}, \mathrm{SU}(2)_{L}, \mathrm{SU}(3)$. The l.h.s. of the first equation is exactly the naive field theory contribution from (MSSM) matter fermions, of $R_{\psi}=Q+\xi / 2$, and gaugino. In the second equation, there are contributions of: $n_{\lambda}$ gauginos of the $\mathrm{SM}$ and $\mathrm{U}(1)_{R}$ gauge groups; gravitino contribution $((-21)$ times that of a gaugino [32]), dilatino and the $\operatorname{Tr}$ is over all matter fermions. These equations agree with the naive field theory result, eq. (B.5), (B.7) for corresponding anomalies. $b_{A, \alpha}$ in the r.h.s. of the first equation also enters in the counterterm due to the GS mechanism in eq. (3.4). By supersymmetry, it shows that the gauge kinetic function coupling $\mathrm{S}$ to all SM sub-groups becomes $k_{\alpha} S$ with $k_{\alpha} \equiv b_{A, \alpha} /\left(12 \pi^{2}\right) .{ }^{17}$ As for the r.h.s. of the second condition in eq. (3.7), in field theory one has $b_{R}=-12 \pi^{2}$, eqs. (B.5), (B.7), while in supergravity one is free to adjust this coefficient (unlike in heterotic string case). This ends the relation of the anomaly cancellation conditions to the naive field theoretical results (global SUSY) obtained using the $\operatorname{Tr}$ over the charged states.

\section{Application to MSSM-like models}

Let us now first apply these anomaly relations to the model of section 2.1. The $\mathrm{U}(1)_{R^{-}}$ charged fermions of the model are the gravitino, gaugino of $\mathrm{U}(1)_{R}$ and dilatino. Their con-

\footnotetext{
${ }^{16}$ For the $\mathrm{U}(1)_{R} \times \mathrm{SM}$ gauge group, $n_{\lambda}=1+(8+3+1)=13$, since the $R$-gaugino contributes, via $\bar{\lambda} \lambda V_{\mu, R}$.

${ }^{17}$ The quantity $b_{A, \alpha} / 12 \pi^{2}=k_{\alpha}$ plays the role of Kac-Moody levels in the heterotic string.
} 
tributions to the cubic $\mathrm{U}(1)_{R}^{3}$ anomaly cancellation of eq. (3.6) give (see also the appendix)

$$
12 \pi^{2} c_{\mathrm{GS}}=3(-\xi / 2)^{3}+(-\xi / 2)^{3}+(\xi / 2)^{3}, \quad \Rightarrow \quad 32 \pi^{2} c_{\mathrm{GS}}=-\left(-b c_{\mathrm{GS}}\right)^{3} .
$$

since in such model $\xi=-b c_{\mathrm{GS}}$. This gives $b>0$, which contradicts our initial assumption $b<0$ needed for the non-perturbative superpotential. Therefore this minimal model is inconsistent at the quantum level and this demands the presence of extra states charged under $\mathrm{U}(1)_{R}$.

Let us then consider a more realistic model. We assume the presence of the MSSM superfields in the visible sector as outlined at the end of section 2.2. For simplicity we assume that all MSSM superfields have $\mathrm{U}(1)_{R}$ charges equal to $Q$, which is indeed possible. ${ }^{18}$ We also allow for the presence of an R-charged superfield $\left(z, \psi_{z}\right)$, singlet under SM group, which contributes only to anomalies that do not involve the SM group.

For the mixed anomalies of $\mathrm{U}(1)_{R}$ with each of the subgroups of the SM: $\mathrm{U}(1)_{Y}$, $\mathrm{SU}(2)_{L}$, $\mathrm{SU}(3)$, we use eq. (3.7) with appropriate generators and obtain, respectively, the three equations below. Using the quantum numbers of the states charged under the SM group: $q:(1 / 6,2,3), u^{c}:(-2 / 3,1, \overline{3}), d^{c}:(1 / 3,1, \overline{3}), l:(-1 / 2,2.1), e^{c}:(1,1,1)$, $\tilde{h}_{1,2}:( \pm 1 / 2,2,1)$ then

$$
\begin{aligned}
11(Q+\xi / 2) & =(1 / 3) c_{\mathrm{GS}} b_{A, 1} \\
7(Q+\xi / 2)-2(\xi / 2) & =(1 / 3) c_{\mathrm{GS}} b_{A, 2} \\
6(Q+\xi / 2)-3(\xi / 2) & =(1 / 3) c_{\mathrm{GS}} b_{A, 3} .
\end{aligned}
$$

To derive eqs. (4.2), we used: $\operatorname{Tr}\left[(Q+\xi / 2) Y_{\psi}^{2}\right]=(Q+\xi / 2) \times 3(1 / 2+1+1 / 6+4 / 3+$ $1 / 3)+1 / 2(1+1)=11(Q+\xi / 2)$ due, in order, to $l, e^{c}, q, u^{c}, d^{c}, \tilde{h}_{1}, \tilde{h}_{2}$; for $\mathrm{SU}(2)_{L}$ : $\operatorname{Tr}\left[(Q+\xi / 2) T^{a} T^{b}\right]=(Q+\xi / 2)[3(1 / 2+3 / 2)+1 / 2(1+1)]=7(Q+\xi / 2)$, from $l, q, \tilde{h}_{1}, \tilde{h}_{2}$. Finally, for $\mathrm{SU}(3): \operatorname{Tr}\left[(Q+\xi / 2) T^{a} T^{b}\right]=(Q+\xi / 2) \times 3(2 \times 1 / 2+1 / 2+1 / 2)=6(Q+\xi / 2)$ from $q, d^{c}, u^{c}$.

The above conditions become

$$
\frac{11(Q+\xi / 2)}{k_{1}}=\frac{7 Q+5(\xi / 2)}{k_{2}}=\frac{6 Q+3(\xi / 2)}{k_{3}}=4 \pi^{2} c_{\mathrm{GS}}, \quad k_{\alpha} \equiv \frac{b_{A, \alpha}}{12 \pi^{2}} .
$$

where $k_{\alpha}$ play the role of Kac-Moody levels, see eq. (3.4) and appendix B. ${ }^{19}$ The cancellation of anomalies thus demands relation (4.3) among the coefficients $b_{A, \alpha}, \alpha: 1,2,3$, which can be tuned to this purpose. This relation is similar to eq. (B.5), (B.7).

By supersymmetry, the three gauge couplings of the SM group are related to coefficients $b_{A, \alpha}$ and the gauge kinetic function becomes $f(S)=k_{\alpha} S$ where $\alpha$ labels $\mathrm{U}(1)_{Y}, \mathrm{SU}(2)_{L}$, $\mathrm{SU}(3)$. Note that we made no assumption about the hypercharge normalization, which is arbitrary. ${ }^{20}$ A GUT-like normalization for it would actually demand the ratios in (4.3) to

\footnotetext{
${ }^{18} \operatorname{Our} \mathrm{U}(1)_{R}$ is realized only locally and we do not consider traditional R-parity symmetry as part of it [11].

${ }^{19}$ The normalized kinetic terms are $1 / 4 \int d^{2} \theta k_{\alpha} S \operatorname{Tr}\left(W^{a} W_{a}\right)_{\alpha}$, with $k_{\alpha}=b_{A, \alpha} /\left(12 \pi^{2}\right)$ as KacMoody levels.

${ }^{20}$ Anomaly cancellation fixes the quantisation of the hypercharge, but not its normalization (such as the $3 / 5$ factor), fixed for example by the presence of a GUT group SU(5), etc, subsequently broken to SM group.
} 
be $b_{A, 1} /(5 / 3)=b_{A, 2}=b_{A, 3}$. In [11] this was attempted for unification purposes and link with the heterotic string theory, etc. However, since it is difficult to derive a gauged $\mathrm{U}(1)_{R}$ from the heterotic string, one may find this too restrictive in some models. Another reason not to impose this demand ${ }^{21}$ is because in such case anomaly cancellation conditions via Green Schwarz are not satisfied [11].

Eqs. (4.3) have implications for the tree level gauge couplings of the SM group which are now fixed by $1 / g_{\alpha}^{2} \equiv k_{\alpha} \operatorname{Re}[s]$. Let us then estimate the values of couplings if $Q=0$ without GUT-like unification conditions. Then, with $\xi=-b c_{\mathrm{GS}}, b<0$ (see sections 2.2 and 2.3$)^{22}$ eq. (4.3) gives positive gauge couplings and $11 / k_{1}=5 / k_{2}=3 / k_{3}=8 \pi^{2} /(-b)$. However, the values of the couplings are not realistic. With $4 \pi / g_{\alpha}^{2}=4 \pi k_{\alpha} \operatorname{Re}[s]=-0.09 \times k_{\alpha} / b$, one finds that $4 \pi / g_{1}^{2} \approx 0.16,4 \pi / g_{2}^{2} \approx 0.07,4 \pi / g_{3}^{2} \approx 0.04$, so all couplings are non-perturbative (for comparison $1 / \alpha_{G U T} \approx 25$ ). Therefore, ${ }^{23}$ one must consider the case with non-zero $Q$ 's for the fermions, which allows a perturbative solution if $C_{\alpha} \equiv \operatorname{Tr}\left[Q \tau^{a} \tau^{b}\right]_{\alpha} \geq \pi|c| /|\operatorname{Re}[s]|$, where $G_{\alpha}$ are the SM sub-groups. mixed $\mathrm{U}(1)_{R}$-SM group anomaly cancellation conditions bring strong constraints. This is so already before considering the $\mathrm{U}(1)_{R}$ cubic and $\mathrm{U}(1)_{R}$ mixed-gravitational anomalies constraints. This ends the discussion about anomalies in which SM subgroups are involved. ${ }^{24}$

Next, let us consider the cubic anomaly of $\mathrm{U}(1)_{R}$. We consider the MSSM spectrum but also include an additional hidden sector state (fermion) $\psi_{z}$ of $\mathrm{U}(1)_{R}$ charge $^{25} R_{z}$, which thus does not affect the discussion so far on anomalies involving the SM group. Then

$$
\begin{aligned}
\operatorname{Tr} R_{\psi}^{3}= & 3\left(2 l^{3}+e^{3}+6 q^{3}+3 u^{3}+3 d^{3}\right)+2\left(\tilde{h}_{1}^{3}+\tilde{h}_{2}^{3}\right)+(8+3+1)(-\xi / 2)^{3} \\
& +3(-\xi / 2)^{3}+(\xi / 2)^{3}+(-\xi / 2)^{3}+R_{z}^{3} \\
= & 49(Q+\xi / 2)^{3}+15(-\xi / 2)^{3}+R_{z}^{3}
\end{aligned}
$$

The first line is due to MSSM matter fermions ${ }^{26}$ and in the second step the $\mathrm{U}(1)_{R}$ charges were replaced by $Q+\xi / 2$. The sum $8+3+1$ is due to gauginos of $\mathrm{SU}(3) \times \mathrm{SU}(2)_{L} \times \mathrm{U}(1)_{Y}$, in this order. In the second line the first term is due to the gravitino (3 times the contribution of a gaugino), the second term is due to dilatino, the third term to $\mathrm{U}(1)_{R}$ gaugino and the last one to the hidden sector $\left(\psi_{z}\right)$. We thus find the following condition from eq. (3.6)

$$
49(Q+\xi / 2)^{3}+15(-\xi / 2)^{3}+R_{z}^{3}=c_{\mathrm{GS}}\left(b_{C}+\xi b_{C K}+\xi^{2} b_{K}\right)
$$

For the mixed $\mathrm{U}(1)_{R}$-gravitational anomaly we have under similar assumptions for the charges

$$
\begin{aligned}
\operatorname{Tr} R_{\psi} & =3\left(2 l+e^{c}+3 u^{c}+3 d^{c}+6 q\right)+2\left(\tilde{h}_{1}+\tilde{h}_{2}\right)+(-\xi / 2)(13-21)+(\xi / 2)+R_{z} \\
& =49(Q+\xi / 2)+9(\xi / 2)+R_{z}
\end{aligned}
$$

\footnotetext{
${ }^{21}$ See [33] for non-standard Kac-Moody levels, and the models with branes at singularities [34-36].

${ }^{22} \xi$ depends on the model, in section $2.1 \xi=-b c_{\mathrm{GS}}$, (see $V_{D}$ ), similar in section 2.2 , also 2.3 if $\beta=0$.

${ }^{23}$ There are examples where one can lift the GUT-like relation and perturbativity and still make predictions, via infrared fixed-point(s) dynamics for ratios of these couplings, which replace the GUT-like constraints [37-39].

${ }^{24}$ The anomaly $\mathrm{U}(1)_{R}^{2}-\mathrm{U}(1)_{Y}$ vanishes, $\operatorname{Tr} Y=0$ on MSSM matter fermions (SM-gravitational anomaly).

${ }^{25}$ Its superpartner is a scalar field $z$, of charge $q_{z}$, see also the discussion in section 2.3 .

${ }^{26}$ Their anomaly contributions are identified by their name in a standard notation.
} 
In the first line $13=(8+3+1)+1$ from all $\mathrm{SM}$ gauginos and $\mathrm{U}(1)_{R}$-gaugino while $+(\xi / 2)$ is due to dilatino. Then

$$
49 Q+29 \xi+R_{z}=-8 c_{\mathrm{GS}} b_{R}
$$

From (4.5), (4.7), with $b_{C}=12 \pi^{2}$, for canonical gauge kinetic term of $\mathrm{U}(1)_{R}$ one must satisfy

$$
\begin{aligned}
R_{z}^{3} & =12 \pi^{2} c_{\mathrm{GS}}\left[1+\xi\left(b_{C K}+\xi b_{K}\right) /\left(12 \pi^{2}\right)\right]-49(Q+\xi / 2)^{3}+15(\xi / 2)^{3} \\
b_{R} & =-\left(29 \xi+R_{z}+49 Q\right) /\left(8 c_{\mathrm{GS}}\right) ;
\end{aligned}
$$

For fixed $Q, \xi, b_{K}$ and $b_{C K}$ one should adjust $R_{z}$ and $b_{R}$ according to eq. (4.8). Thus, canceling the cubic anomaly is possible by adding $\psi_{z}$ of freely adjustable $R_{\psi}$, even if $b_{K}=b_{C K}=0$. This condition can impact on the existence or stability of the ground state of the model. Indeed, the scalar $z$ superpartner of $\psi_{z}$ participates in the minimisation conditions of the scalar potential that fixes the ground state; these may impose restrictions on $q_{z}=R_{\psi}-\xi / 2$ (such as its sign) inconsistent with the above result for $R_{\psi} \cdot{ }^{27}$ These can alter the previous predictions for $m_{3 / 2} \sim \mathrm{TeV}$. One can avoid this case by tuning $b_{K}$ or $b_{C K}$ and $b_{R}$ to respect (4.8), see eq. (3.4).

Case (a): no extra state $\left(\boldsymbol{z}, \boldsymbol{\psi}_{\boldsymbol{z}}\right)$. What happens if no extra hidden state $\psi_{z}$ is present? From eq. (4.8) with $Q=b_{C K}=b_{K}=0$ we have $12 \pi^{2} c_{\mathrm{GS}}=34\left(-b c_{\mathrm{GS}} / 2\right)^{3}$ giving $^{28}$

$$
\left|m_{3 / 2}\right|=\kappa^{-1}|a b / \alpha| e^{\alpha} \sim \kappa^{-1}|b|^{3 / 2} c_{\mathrm{GS}} e^{\alpha} /|\alpha| \sim\left(48 \pi^{2} / 17\right)^{\frac{1}{2}} \kappa^{-1} e^{\alpha / 2} /|\alpha| .
$$

Therefore, the gravitino mass becomes of the order of Planck scale and is not "tunable" anymore to a $\mathrm{TeV}$ value. As a result, the soft terms masses would also become of the order of the Planck scale. The reason for this result is that the GS mechanism (related to $\sim c_{\mathrm{GS}}$ ) and anomaly cancellation in the presence of FI terms (related to $\xi \sim b c_{\mathrm{GS}}$ ), when put together are too restrictive given the minimal field content in the hidden sector.

Case (b): including the state $(\boldsymbol{z}, \boldsymbol{\psi})$. This is similar to the model of section 2.3 "upgraded" in the visible sector by the MSSM superfields. These do not alter the discussion there regarding the ground state, etc, since the MSSM scalars are $\mathrm{U}(1)_{R}$ neutral if we set their $Q=0$. Condition (4.8) for the cubic anomaly can be re-written as follows (with $\left.b_{C K}=b_{K}=0\right)$ :

$$
\left(q_{z}-b c_{\mathrm{GS}} / 2\right)^{3}=12 \pi^{2} c_{\mathrm{GS}}+34\left(b c_{\mathrm{GS}} / 2\right)^{3}
$$

using that $R_{z}=q_{z}+\xi / 2$ with $q_{z}$ the charge of the scalar superpartner of $\psi_{z}$ and $\xi=-b c_{\mathrm{GS}}$.

We found in section 2.3 that if $q_{z}<0, c_{\mathrm{GS}}>0$ one preserves the usual ground state and that $c_{\mathrm{GS}}$ is numerically very small $c_{\mathrm{GS}} \sim 10^{-13}, b \sim \mathcal{O}\left(10^{-3}\right)$ for a $\mathrm{TeV}$ gravitino mass (see section 2). This means that we can ignore the last term in the r.h.s. of eq. (4.10). Then the above equation has no solution $q_{z}<0$. Therefore, while one can always add hidden sector states to cancel anomalies, the result is that the ground state is modified so the prediction $m_{3 / 2} \sim \mathrm{TeV}$ is not valid anymore ${ }^{29}$ and the phenomenological motivation is lost.

\footnotetext{
${ }^{27}$ Recall the constraints $q_{z}<0$ and $c_{\mathrm{GS}}>0$ in section 2.3 , see also case (b) later on.

${ }^{28}$ We set $Q=0$ since with $\mathrm{U}(1)_{R}$ charged MSSM scalar fields, this value of $m_{3 / 2}$ is not valid anymore.

${ }^{29}$ In section 2.3, the gravitino mass was $\left|m_{3 / 2}\right|=\left|\kappa^{2} e^{\kappa^{2} \mathcal{K} / 2} W\right|=\kappa^{-1}|a b / \alpha| e^{\alpha} \sim \kappa^{-1}(-b)^{3 / 2} c_{\mathrm{GS}} e^{\alpha} /|\alpha|$.
} 
There is in principle one option left: use either $b_{K}$ and/or $b_{C K}$ to enforce $q_{z}<0$ and maintain the $\mathrm{TeV}$-value of $m_{3 / 2}$. This option can also be used for the minimal model in section 2.1 to relax its cubic anomaly constraint in eq. (4.1). It is also possible that when adding more fields in the hidden sector of different charges, the anomalies cancel without changing the ground state, with $m_{3 / 2} \sim \mathrm{TeV}$. But then finding the ground state and its properties become difficult tasks.

To conclude, anomaly cancellation in the presence of FI terms and $\mathrm{U}(1)_{R}$ gauge symmetry, even in the presence of a Green-Schwarz mechanism and after relaxing the GUTlike constraints for tree level gauge couplings, is a very restrictive constraint for model building (MSSM-like models). In an anomaly-free model, a tunable, TeV-scale gravitino mass may remain possible provided that the $\mathrm{U}(1)_{R}$ charges of additional hidden sector fermions (constrained by anomalies) do not conflict with the related values of the $\mathrm{U}(1)_{R}$ charges of their scalar superpartners, constrained by existence of a stable ground state. This issue may be bypassed by tuning instead the coefficients of the Kahler connection anomalies $\left(b_{K}, b_{C K}\right){ }^{30}$

\section{Conclusions}

In this work we analyzed, at the classical level, some models with a shift symmetry of the dilaton that is gauged into a $\mathrm{U}(1)_{R}$ symmetry in $4 \mathrm{D} N=1$ supergravity. We then studied the impact of quantum constraints such as anomaly cancellation on these models.

At the classical level, a gauged $\mathrm{U}(1)_{R}$ symmetry dictates the structure of the superpotential $W \sim e^{b s}$ where $s$ is the dilaton which transforms non-linearly under $\mathrm{U}(1)_{R}$. With a minimal supergravity spectrum containing the dilaton (sgoldstino), gravitino, dilatino, massive $\mathrm{U}(1)_{R}$ gauge boson and its R-gaugino superpartner, such a toy model can have spontaneous breaking of supersymmetry with a (small, positive) tunable cosmological constant, TeV-gravitino mass and gravity-mediation scale.

We showed that these nice properties can be maintained in the presence of additional states in the visible and hidden sectors, under some minimal assumptions. The visible sector can be that of the MSSM if its chiral superfields are considered R-neutral. This means that fermions have $\mathrm{U}(1)_{R}$ charges of order $\xi \sim b c_{\mathrm{GS}}$, where $\xi$ is the Fayet-Iliopoulos constant and $c_{\mathrm{GS}}$ is the shift of the axion, Im $[s]$. Additional R-charged field(s) in the hidden sector can be present and still maintain $m_{3 / 2} \sim \mathrm{TeV}$, under some constraints for the R-charge(s).

At the quantum level, we examined the anomaly cancellation conditions in supergravity with a gauge group of $\mathrm{SM} \times \mathrm{U}(1)_{R}$. The spectrum is that of minimal $4 \mathrm{D} \mathrm{N}=1$ supergravity

\footnotetext{
${ }^{30}$ There is a possible correction to our analysis that may be worth investigating. The presence of a nontrivial dilaton Kahler potential $\mathcal{K} \sim-2 \ln (s+\bar{s})$ lead to a non-flat field space metric $\mathcal{K}_{s}^{s}=\partial^{2} \mathcal{K} / \partial s \partial s^{\dagger} \sim$ $2 /(s+\bar{s})^{2}$. The anomaly cancellation conditions eqs. (3.1), which lead to the familiar field theory condition on the R-charges (eq. (3.7)) of cubic anomaly cancellation, does not take into account the effect of this non-flat metric. This effect impacts on anomaly cancellation via a tensor $\Sigma_{\mu \nu s}^{s}=1 /(s+\bar{s})^{2} \times\left(D_{\mu} s D_{\nu} \bar{s}-D_{\nu} s D_{\mu} \bar{s}\right)$, which "mixes" the space-time indices with the field indices. This tensor is just the target space curvature tensor "pulled back" to space-time and is present in the covariant derivatives of the fermions. In principle, the formalism in $[22,23]$ could be applied to see if the non-flat metric impacts on the cubic and the other anomalies cancellation.
} 
extended by MSSM-like superfields also charged under $\mathrm{U}(1)_{R}$. Cubic and mixed anomalies of an anomalous U(1) with the SM gauge group, Kahler connection and gravity were studied in the past in a general approach in gauged supergravity with Green-Schwarz mechanism and FI terms. We showed the agreement of these anomaly cancellation conditions (other than that involving Kahler connection) to the "naive" field theory approach in global SUSY (using Tr over charges and GS mechanism) for the special case of gauged $\mathrm{U}(1)_{R}$, with Rcharges determined using simple arguments (appendix A). Note that in global SUSY U $(1)_{R}$ cannot be gauged and the $\mathrm{U}(1)_{R}$ charges depend on the FI terms.

We then applied the anomaly conditions to the MSSM as visible sector, with superfields of $\mathrm{U}(1)_{R^{-}}$-charges equal to $Q$ while fermions charges are shifted by the FI term, and relaxed the GUT-like unification condition. Even after doing so, the $\mathrm{U}(1)_{R^{-}} \mathrm{SM}$ mixed-anomalies cancellation remains a strong parametric constraint that impacts on perturbativity of the gauge couplings $(Q=0)$. Even without these constraints, the cancellation of the $\mathrm{U}(1)_{R}$ cubic anomaly on its own brings constraints on the gravitino mass (and thus soft terms masses) which becomes of the order of Planck scale. The addition to the hidden sector

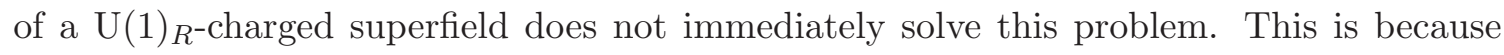
the anomaly-induced constraint on the R-charge of the scalar component can modify the ground state of the model, thus loosing its properties like $m_{3 / 2} \sim \mathrm{TeV}$. A possible tuning of the coefficients $\left(b_{K}, b_{C K}\right)$ of the Kahler connection anomalies may bypass this problem. Alternatively a more complicated hidden sector could avoid this issue, but it makes very difficult an analysis of the existence of the ground state, with $m_{3 / 2} \sim \mathrm{TeV}$.

\section{Acknowledgments}

The work of D. Ghilencea was supported by a grant of the Romanian National Authority for Scientific Research, CNCS-UEFISCDI, project number PN-II-ID-PCE-2011-3-0607. D.G. thanks Hyun Min Lee (Chung-Ang University, Seoul) for interesting discussions on this topic.

\section{A Gauged $\mathrm{U}(1)_{R}$ and fields charges}

The action considered is

$$
\begin{aligned}
\mathcal{S} & =\int d^{4} x\left\{d^{4} \theta \mathbf{E}\left[\left(-3 / \kappa^{2}\right) S_{0}^{\dagger} e^{2(\xi / 3) V_{R}} S_{0} e^{-\kappa^{2} K_{0} / 3}\right]+\left[\int d^{2} \theta \mathcal{E} S_{0}^{3} W\left(\Phi^{i}\right)+\text { h.c. }\right]\right\} \\
\kappa^{2} K & \equiv \kappa^{2} K_{0}-2 \xi V_{R}
\end{aligned}
$$

$\Phi^{i}$ are matter superfields charged or not under these groups. $S_{0}$ is the conformal compensator superfield, $\mathbf{E}$ is the superspace measure, $\mathcal{E}$ is the chiral superspace measure. $V_{R}$ is a $\mathrm{U}(1)_{R}$ vector superfield, $\xi$ is the constant Fayet-Iliopoulos term. In the flat limit $\mathcal{S} \supset \int d^{4} \theta\left(K_{0}-2 \xi V_{R}\right)$. If $K_{0} \supset \Phi^{\dagger} \exp \left(2 q V_{R}\right) \Phi$ then $\mathcal{S} \supset q|\phi|^{2}-\xi D+D^{2} / 2$ giving $D^{2} \sim\left(q|\phi|^{2}-\xi\right)^{2}$. The action is invariant under a super-Weyl symmetry (eq. (2.9) in [40])

$$
\begin{aligned}
& \lambda \rightarrow e^{-3 \tau} \lambda \quad \mathbf{E} \rightarrow e^{2 \tau+2 \bar{\tau}} \mathbf{E} \quad \mathcal{E} \rightarrow e^{6 \tau} \mathcal{E} \quad \mathcal{W}_{\alpha} \rightarrow e^{-3 \tau} \mathcal{W}_{\alpha}, \\
& V^{(a)} \rightarrow V^{(a)} \quad S_{0} \rightarrow e^{-2 \tau} S_{0} \quad W \rightarrow W \quad \overline{\mathcal{W}}^{\dot{\alpha}} \rightarrow e^{-3 \bar{\tau}} \overline{\mathcal{W}}^{\dot{\alpha}}
\end{aligned}
$$


with complex parameter $\tau$. Note that the superpotential does not transform, while in our case it does (so super-Weyl transformation is a particular case of an $R$-symmetry). We thus need an extra $\mathrm{U}(1)_{R}$; under a $\mathrm{U}(1)_{R}$ gauge transformation ${ }^{31}$

$$
V_{R} \rightarrow V_{R}+\frac{i}{2}\left(\Lambda-\Lambda^{\dagger}\right), \quad \bar{D} \Lambda=0
$$

$K$ must then transform ( $K_{0}$ invariant)

$$
\kappa^{2} K \rightarrow \kappa^{2} K-i \xi\left(\Lambda-\Lambda^{\dagger}\right)
$$

The action is invariant under $\mathrm{U}(1)_{R}$ if

$$
S_{0} \rightarrow S_{0}^{\prime}=e^{-i \xi / 3 \Lambda} S_{0}, \quad W \rightarrow W^{\prime}=e^{+i \xi \Lambda} W \quad \Rightarrow \quad R_{W}=-\xi .
$$

and $G=\kappa^{2} K+\ln \left|W \kappa^{3}\right|^{2}$ is invariant, too. ${ }^{32}$ The choice of super-Weyl gauge $S_{0}=s_{0}+\theta^{2} F$ can be maintained if we combine the previous super-Weyl and the $\mathrm{U}(1)_{R}$ transformations such as the conformal compensator remains invariant (neutral). This is possible provided that [14]

$$
-2 \tau-i(\xi / 3) \Lambda=0, \quad \rightarrow \quad \tau=-\frac{i \xi \Lambda}{6} .
$$

thus $R_{\lambda}=R_{\mathcal{W}}=-\xi / 2$ according to (A.2). We thus work in this gauge which keeps manifest SUSY and holomorphicity and the compensator is neutral under this $\mathrm{U}(1)_{R}$. A consequence is that, according to the transformation of $\mathbf{E}$, the gravitino will carry a charge under this new $\mathrm{U}(1)_{R}$. Further, we also have

$$
R_{V}=0, \quad R_{\mathcal{W}}=-\frac{\xi}{2}=R_{\bar{D}^{2} D}
$$

With $V \supset \overline{\theta \theta} \theta \lambda$ then $R_{\theta}=R_{\lambda}=-\xi / 2$. A superfield of R-charge $Q$ transforms as $\Phi \rightarrow$ $e^{-i Q \Lambda} \Phi$.

The component form of the action contains, for a superfield $\Phi=\left(\phi, \psi_{\phi}\right)[20]$

$$
L \supset X^{\phi} \bar{\psi}_{\phi} \bar{\lambda}+\text { h.c. }
$$

$X^{\phi}$ is the Killing vector of scalar $\phi$ and $\lambda$ the R-gaugino. For a superfield with $\Phi \rightarrow e^{-i Q \Lambda} \Phi$

$$
X^{\phi} \propto i \phi \quad \Rightarrow \quad R_{\psi_{\phi}}=R_{\phi}-R_{\lambda}=Q+\frac{\xi}{2}
$$

which was used in the text. Consider now that $K_{0}$ contains a dilaton $(S)$ dependent term

$$
K_{0} \supset-\ln \left(S+S^{\dagger}-\delta V_{R}\right)
$$

\footnotetext{
${ }^{31}$ This transformation on $V_{R}$ corresponds to a gauge transformation $A_{\mu} \rightarrow A_{\mu}+\partial_{\mu} \rho$ where $\rho=\operatorname{Re} \Lambda_{\theta=\bar{\theta}=0}$. Under this gauge transformation, the scalar fields transform as: $\phi \rightarrow \exp (-i q \rho) \phi$, with $D_{\mu} \phi \equiv\left(\partial_{\mu} \phi+\right.$ $\left.\operatorname{ig} q A_{\mu}\right) \phi$ where $q$ is the charge of the field $\phi$. This is consistent with conventions in the text: $D_{\mu} \phi^{j} \equiv$ $\partial_{\mu} \phi^{j}-X^{j}(\phi) A_{\mu}$, and $\delta \phi^{j}=\rho X^{j}(\phi)$ and the Killing vectors $X^{j}(\phi)=-i q \phi^{j}$ for a linearly transformed $\phi$. These conventions are similar to those in [18] but charges $q$ and $\xi$ of opposite signs.

${ }^{32}$ With $W=a e^{b S},(b<0)$, under transformation $S \rightarrow S-i c_{\mathrm{GS}} \Lambda$ gives $\xi=-b c_{\mathrm{GS}}$ and $D^{2} \sim\left(q|\phi|^{2}+\right.$ $\left.b c_{\mathrm{GS}}\right)^{2}$.
} 
which is invariant under our gauged $\mathrm{U}(1)_{R}$ provided

$$
S \rightarrow S+i \frac{\delta}{2} \Lambda, \quad \delta \text { real; } \quad s \rightarrow s+i \frac{\delta}{2} \rho,\left.\quad \rho \equiv(\operatorname{Re} \Lambda)\right|_{\theta=\bar{\theta}=0}
$$

For the dilaton $S=\left(s, \psi_{s}\right)$

$$
X^{s}=+i \delta, \quad \Rightarrow \quad R_{\psi_{s}}=-R_{\lambda}=\frac{\xi}{2} \quad \Rightarrow \quad R_{\phi^{s}}=R_{\psi_{s}}+R_{\theta}=0
$$

Regarding the gravitino $\psi_{(3 / 2)}$, from any of the terms of the supergravity Lagrangian

$$
L \supset-\frac{1}{2} e D_{a} \psi_{(3 / 2)} \sigma \bar{\lambda}^{a}+e^{K / 2} W \bar{\psi}_{(3 / 2)} \sigma \bar{\psi}_{(3 / 2)}+\text { h.c. }
$$

one obtains the value of $R_{\psi_{3 / 2}}$ used in the text:

$$
R_{\psi_{(3 / 2)}}=R_{\lambda}=\frac{1}{2} R_{W}=\frac{-\xi}{2} .
$$

\section{B Cancellation of anomalies with a $\mathrm{U}(1)_{R} \times \mathrm{SM}$ group}

Consider the Lagrangian in the global SUSY limit, with S the dilaton:

$$
\begin{aligned}
\mathcal{L}= & -\int d^{4} \theta \ln \left(S+\bar{S}-\delta V_{R}\right) \\
& +\left\{\frac{1}{16 \pi^{2} \kappa} \int d^{2} \theta\left[k_{R} S \mathcal{W}_{R}^{\alpha} \mathcal{W}_{R, \alpha}+k_{a} S \operatorname{Tr} \mathcal{W}_{a}^{\alpha} \mathcal{W}_{a, \alpha}\right]+\text { h.c. }\right\}
\end{aligned}
$$

where $\kappa$ cancels the Tr factor in non-Abelian case. $k_{R}$ and $k_{a}$ are Kac-Moody levels of $\mathrm{U}(1)_{R}$ and subgroups $\left.G_{a}: \mathrm{U}(1)_{Y}, S U 2\right)_{L}, \mathrm{SU}(3)$ of the SM group. For example for $\mathrm{U}(1)_{R}$ part

$$
\begin{aligned}
\mathcal{L} & \supset \frac{1}{16 g^{2}} \int d^{2} \theta k_{R} S \mathcal{W}_{R}^{2} \\
& \supset k_{R}\left\{\frac{-1}{4} \operatorname{Re}[s] C_{\mu \nu} C^{\mu \nu}+\frac{1}{2} \operatorname{Re}[s] D_{a}^{2}+\frac{1}{4} \operatorname{Im}[s] C_{\mu \nu} \tilde{C}^{\mu \nu}\right\}
\end{aligned}
$$

where $\tilde{C}^{\mu \nu}=1 / 2 \epsilon^{\mu \nu \rho \sigma} C_{\rho \sigma}$. Consider the shift

$$
S \rightarrow S+i \frac{\delta}{2} \Lambda(x)
$$

and define

$$
C_{\alpha}=\operatorname{Tr}_{G_{\alpha}}\left[T(r)^{2} R_{\psi}\right]
$$

$C_{\alpha}$ denotes the mixed anomaly $\mathrm{U}(1)_{R}$ with $G_{\alpha}=\mathrm{U}(1)_{Y}, \mathrm{SU}(2)_{L}$, or $\mathrm{SU}(3)$ and $R_{\psi}$ is the $\mathrm{U}(1)_{R}$ charge with fermions transforming as $\psi \rightarrow \exp \left(-i R_{\psi} \rho\right) \psi$ with $\rho=\left.\operatorname{Re} \Lambda\right|_{\theta=\bar{\theta}=0} . R_{\psi}$ depend on the Fayet-Iliopoulos constant $(\xi)$ as discussed in previous appendix.

The anomalous $\mathrm{U}(1)_{R}$ generates $\Delta \mathcal{L} \propto \int d^{2} \theta\left[i C_{\alpha} \Lambda \mathcal{W}_{a} \mathcal{W}_{a}\right]$ for each subgroup $G_{\alpha}$. The shift of the dilaton is the same for all $G_{\alpha}$ (ignoring $k_{\alpha}$ ) then the ratio $C_{\alpha} / k_{\alpha}$ must be 
identical for all $G_{\alpha}$ for anomalies to cancel. Also taking the $\mathrm{U}(1)_{R}^{3}$ and $\mathrm{U}(1)_{R}$-gravitational anomaly, then one has the result

$$
-4 \pi^{2} \delta=\frac{2 C_{\alpha}}{k_{\alpha}}=\frac{(2 / 3) \operatorname{Tr} R_{\psi}^{3}}{k_{R}}=\frac{1}{12} \operatorname{Tr} R_{\psi},
$$

In the paper we kept $k_{R}=1$. The gauge couplings constants are then $k_{\alpha}\langle\operatorname{Re}[s]\rangle \equiv 1 / g_{\alpha}^{2}$.

Regarding the mixed gravitational anomaly (last term above) this is seen from the action

$$
L \supset \frac{-1}{4} \operatorname{Im}[s] \mathcal{R} \tilde{\mathcal{R}}, \quad \Delta L_{\text {axion }}=-\frac{\rho}{8} \delta \mathcal{R} \tilde{\mathcal{R}}, \quad \Delta L_{\text {anomaly }}=\frac{\rho}{384 \pi^{2}} \operatorname{Tr}\left[R_{\psi}\right] \mathcal{R} \tilde{\mathcal{R}}
$$

and use $\Delta L_{\text {axion }}+\Delta L_{\text {anomaly }}=0$. However, unlike in heterotic string, in supergravity, $\mathcal{R} \tilde{\mathcal{R}}$ term can have a different coefficient from $\operatorname{Im}[s]$ since it is not part of the leading order action.

In the text we used the notation $W=a e^{b S}$, with $b<0$ and $S \rightarrow S-i c_{\mathrm{GS}} \Lambda$ giving $\delta / 2=-c_{\mathrm{GS}}$. The anomaly condition (B.5) becomes

$$
4 \pi^{2} c_{\mathrm{GS}}=\frac{C_{\alpha}}{k_{\alpha}}=\frac{(1 / 3) \operatorname{Tr} R_{\psi}^{3}}{k_{R}}=\frac{1}{24} \operatorname{Tr}\left[R_{\psi}\right]
$$

This result was compared against the more general supergravity results in section 3 .

Open Access. This article is distributed under the terms of the Creative Commons Attribution License (CC-BY 4.0), which permits any use, distribution and reproduction in any medium, provided the original author(s) and source are credited.

\section{References}

[1] A. Maloney, E. Silverstein and A. Strominger, De Sitter space in noncritical string theory, hep-th/0205316 [INSPIRE].

[2] C.P. Burgess, R. Kallosh and F. Quevedo, De Sitter string vacua from supersymmetric D terms, JHEP 10 (2003) 056 [hep-th/0309187] [INSPIRE].

[3] S. Kachru, R. Kallosh, A.D. Linde and S.P. Trivedi, De Sitter vacua in string theory, Phys. Rev. D 68 (2003) 046005 [hep-th/0301240] [INSPIRE].

[4] K. Choi, A. Falkowski, H.P. Nilles, M. Olechowski and S. Pokorski, Stability of flux compactifications and the pattern of supersymmetry breaking, JHEP 11 (2004) 076 [hep-th/0411066] [INSPIRE].

[5] K. Choi, K.S. Jeong and K.-i. Okumura, Phenomenology of mixed modulus-anomaly mediation in fluxed string compactifications and brane models, JHEP 09 (2005) 039 [hep-ph/0504037] [INSPIRE].

[6] K. Choi, A. Falkowski, H.P. Nilles and M. Olechowski, Soft supersymmetry breaking in KKLT flux compactification, Nucl. Phys. B 718 (2005) 113 [hep-th/0503216] [INSPIRE].

[7] M. Endo, M. Yamaguchi and K. Yoshioka, A bottom-up approach to moduli dynamics in heavy gravitino scenario: superpotential, soft terms and sparticle mass spectrum, Phys. Rev. D 72 (2005) 015004 [hep-ph/0504036] [INSPIRE]. 
[8] O. Lebedev, H.P. Nilles and M. Ratz, De Sitter vacua from matter superpotentials, Phys. Lett. B 636 (2006) 126 [hep-th/0603047] [INSPIRE].

[9] T. Banks, Supersymmetry breaking and the cosmological constant, Int. J. Mod. Phys. A 29 (2014) 1430010 [arXiv:1402.0828] [InSPIRE].

[10] E. Cremmer, S. Ferrara, L. Girardello, C. Kounnas and A. Masiero, Superhiggs effect with local $R$ symmetry and vanishing cosmological constant, Phys. Lett. B 137 (1984) 62 [INSPIRE].

[11] A.H. Chamseddine and H.K. Dreiner, Anomaly free gauged R symmetry in local supersymmetry, Nucl. Phys. B 458 (1996) 65 [hep-ph/9504337] [INSPIRE].

[12] D.J. Castano, D.Z. Freedman and C. Manuel, Consequences of supergravity with gauged $\mathrm{U}(1)_{R}$ symmetry, Nucl. Phys. B 461 (1996) 50 [hep-ph/9507397] [INSPIRE].

[13] P. Binetruy, G. Dvali, R. Kallosh and A. Van Proeyen, Fayet-Iliopoulos terms in supergravity and cosmology, Class. Quant. Grav. 21 (2004) 3137 [hep-th/0402046] [INSPIRE].

[14] H.M. Lee, Supersymmetric codimension-two branes and $\mathrm{U}(1)_{R}$ mediation in $6 D$ gauged supergravity, JHEP 05 (2008) 028 [arXiv:0803.2683] [INSPIRE].

[15] I. Antoniadis, J.-P. Derendinger and T. Maillard, Nonlinear $N=2$ supersymmetry, effective actions and moduli stabilization, Nucl. Phys. B 808 (2009) 53 [arXiv:0804.1738] [INSPIRE].

[16] Y. Kawamura and T. Kobayashi, Soft scalar masses in string models with anomalous $\mathrm{U}(1)$ symmetry, Phys. Lett. B 375 (1996) 141 [Erratum ibid. B 388 (1996) 867] [hep-ph/9601365] [INSPIRE].

[17] Y. Kawamura and T. Kobayashi, Generic formula of soft scalar masses in string models, Phys. Rev. D 56 (1997) 3844 [hep-ph/9608233] [INSPIRE].

[18] G. Villadoro and F. Zwirner, De-Sitter vacua via consistent D-terms, Phys. Rev. Lett. 95 (2005) 231602 [hep-th/0508167] [INSPIRE].

[19] I. Antoniadis and R. Knoops, Gauge R-symmetry and de Sitter vacua in supergravity and string theory, Nucl. Phys. B 886 (2014) 43 [arXiv: 1403.1534] [INSPIRE].

[20] J. Wess and J. Bagger, Supersymmetry and supergravity, $2^{\text {nd }}$ edition, Princeton University Press, Princeton, U.S.A. (1992).

[21] D. Freedman and A. Van Proeyen, Supergravity, Cambridge University Press, Cambridge U.K. (2012), see chapter 18.

[22] H. Elvang, D.Z. Freedman and B. Körs, Anomaly cancellation in supergravity with Fayet-Iliopoulos couplings, JHEP 11 (2006) 068 [hep-th/0606012] [INSPIRE].

[23] D.Z. Freedman and B. Körs, Kähler anomalies in supergravity and flux vacua, JHEP 11 (2006) 067 [hep-th/0509217] [INSPIRE].

[24] P. Anastasopoulos, M. Bianchi, E. Dudas and E. Kiritsis, Anomalies, anomalous U(1)'s and generalized Chern-Simons terms, JHEP 11 (2006) 057 [hep-th/0605225] [INSPIRE].

[25] P. Binetruy and E. Dudas, Gaugino condensation and the anomalous U(1), Phys. Lett. B 389 (1996) 503 [hep-th/9607172] [InSPIRE].

[26] P. West, Introduction to supersymmetry and supergravity, World Scientific, Singapore (1986).

[27] F. Catino, G. Villadoro and F. Zwirner, On Fayet-Iliopoulos terms and de Sitter vacua in supergravity: some easy pieces, JHEP 01 (2012) 002 [arXiv:1110.2174] [INSPIRE]. 
[28] S.R. Coleman, V. Glaser and A. Martin, Action minima among solutions to a class of euclidean scalar field equations, Commun. Math. Phys. 58 (1978) 211 [InSPIRE].

[29] S.R. Coleman, The fate of the false vacuum. 1. Semiclassical theory, Phys. Rev. D 15 (1977) 2929 [Erratum ibid. D 16 (1977) 1248] [INSPIRE].

[30] M.J. Duncan and L.G. Jensen, Exact tunneling solutions in scalar field theory, Phys. Lett. B 291 (1992) 109 [INSPIRE].

[31] K. Blum, C. Delaunay and Y. Hochberg, Vacuum (meta)stability beyond the MSSM, Phys. Rev. D 80 (2009) 075004 [arXiv:0905.1701] [INSPIRE].

[32] N.K. Nielsen, M.T. Grisaru, H. Romer and P. van Nieuwenhuizen, Approaches to the gravitational spin $3 / 2$ axial anomaly, Nucl. Phys. B 140 (1978) 477 [INSPIRE].

[33] K.R. Dienes, A.E. Faraggi and J. March-Russell, String unification, higher level gauge symmetries and exotic hypercharge normalizations, Nucl. Phys. B 467 (1996) 44 [hep-th/9510223] [INSPIRE].

[34] G. Aldazabal, L.E. Ibáñez and F. Quevedo, A D-brane alternative to the MSSM, JHEP 02 (2000) 015 [hep-ph/0001083] [INSPIRE].

[35] G. Aldazabal, L.E. Ibáñez, F. Quevedo and A.M. Uranga, D-branes at singularities: a bottom up approach to the string embedding of the standard model, JHEP 08 (2000) 002 [hep-th/0005067] [INSPIRE].

[36] D.M. Ghilencea, A note on two loop effects in the DMSSM, Nucl. Phys. B 622 (2002) 215 [hep-ph/0110008] [INSPIRE].

[37] D. Ghilencea, M. Lanzagorta and G.G. Ross, Strong unification, Phys. Lett. B 415 (1997) 253 [hep-ph/9707462] [INSPIRE].

[38] G. Amelino-Camelia, D. Ghilencea and G.G. Ross, The effect of Yukawa couplings on unification predictions and the nonperturbative limit, Nucl. Phys. B 528 (1998) 35 [hep-ph/9804437] [INSPIRE].

[39] D. Ghilencea, M. Lanzagorta and G.G. Ross, Unification predictions, Nucl. Phys. B 511 (1998) 3 [hep-ph/9707401] [INSPIRE].

[40] V. Kaplunovsky and J. Louis, Field dependent gauge couplings in locally supersymmetric effective quantum field theories, Nucl. Phys. B 422 (1994) 57 [hep-th/9402005] [INSPIRE]. 